\title{
mTOR signaling promotes foam cell formation and inhibits foam cell egress through suppressing the SIRT1 signaling pathway
}

\author{
HAIXIANG ZHENG ${ }^{1}$, YUCAI FU ${ }^{2}$, YUSHENG HUANG ${ }^{1}$, XINDE ZHENG ${ }^{1}$, WEI YU ${ }^{1}$ and WEI WANG ${ }^{1}$ \\ ${ }^{1}$ Department of Cardiology, The Second Affiliated Hospital of Shantou University Medical College; \\ ${ }^{2}$ Laboratory of Cell Senescence, Shantou University Medical College, Shantou, Guangdong 515041, P.R. China
}

Received February 28, 2017; Accepted July 7, 2017

DOI: $10.3892 / \mathrm{mmr} .2017 .7032$

\begin{abstract}
Atherosclerosis (AS) is a chronic immuno-inflammatory disease accompanied by dyslipidemia. The authors previously demonstrated that sirtuin 1 (SIRT1) may prevent atherogenesis through influencing the liver $\mathrm{X}$ receptor/C-C chemokine receptor type 7/nuclear factor- $\kappa \mathrm{B}$ (LXR-CCR7/NF-kB) signaling pathway. Previous studies have suggested a role for mammalian target of rapamycin (mTOR) signaling in the pathogenesis of cardiovascular diseases. The present study investigated the potential association between mTOR signaling and SIRT1-LXR-CCR7/NF- $\mathrm{KB}$ signaling (SIRT1 signaling) in AS pathogenesis. To induce foam cell formation, U937 cells were differentiated into macrophages by exposure to phorbol 12-myristate 13-acetate (PMA) for $24 \mathrm{~h}$, followed by treatment with palmitate and oxidized low density lipoprotein for a further $24 \mathrm{~h}$. Oil red $\mathrm{O}$ staining revealed a large accumulation of lipid droplets present in foam cells. Western blot analysis demonstrated increased protein levels of phosphorylated (p)-mTOR and its downstream factor p-ribosomal protein S6 kinase (p70S6K). Reverse transcription-quantitative
\end{abstract}

Correspondence to: Wei Wang, Department of Cardiology, The Second Affiliated Hospital of Shantou University Medical College, 69 Dongxia North Road, Shantou, Guangdong 515041, P.R. China

E-mail: wangwei_sumc@126.com

Abbreviations: AS, atherosclerosis; SIRT1, sirtuin 1; LXR, liver $\mathrm{X}$ receptor; CCR7, C-C chemokine receptor type 7; NF- $\kappa \mathrm{B}$, nuclear factor- $\kappa \mathrm{B}$; mTOR, mammalian target of rapamycin; PMA, phorbol 12-myristate 13-acetate; TNF- $\alpha$, tumor necrosis factor- $\alpha$; LPA, lysophosphatidic acid; ABCA1, adenosine triphosphate-binding cassette transporter A1; APC, antigen-presenting cells; DCs, dendritic cells; ox-LDL, oxidized low density lipoprotein; DAPI, 4'6-diamidino-2-phenylindole dihydrochloride; p70S6K, p-ribosomal protein S6 kinase; RT, room temperature; MTT, 3-(4,5-dimethylthiazol-2-yl)-2,5-diphenyltetrazolium; SDS, sodium dodecyl sulfate; BSA, bovine serum albumin; GAPDH, glyceraldehyde 3-phosphate dehydrogenase; TBS, Tris-buffered saline

Key words: atherosclerosis, mTOR, SIRT1 signaling, monocyte-derived foam cell polymerase chain reaction and western blot analyses additionally revealed decreased expression of SIRT1, LXR $\alpha$ and CCR7 and increased expression of $\mathrm{NF}-\kappa \mathrm{B}$ and its downstream factor tumor necrosis factor- $\alpha(\mathrm{TNF}-\alpha)$ in an atherogenetic condition induced by lysophosphatidic acid (LPA). In addition, abundant lipid droplets accumulated in U937-LPA-treated foam cells. Rapamycin, an mTOR inhibitor, suppressed the expression and activity of mTOR and p70S6K, however enhanced expression of SIRT1, LXR $\alpha$, and CCR7. Conversely, rapamycin deceased TNF- $\alpha$ and NF- $\kappa$ B activity, the latter of which was further confirmed by immunofluorescence analysis demonstrating increased levels of NF- $\mathrm{B}$ present in the cytoplasm compared with the nucleus. The findings of the present study suggest that mTOR signaling promotes foam cell formation and inhibits foam cell egress via suppression of SIRT1 signaling.

\section{Introduction}

Atherosclerotic arterial disease is one of the leading causes of morbidity, mortality and disability worldwide, and the prevalence of atherosclerosis (AS) is increasing in developing countries $(1,2)$. AS is initiated by circulating monocytes infiltrating the subendothelial space in response to multiple stimuli, such as hyperlipidemia, and then differentiating into macrophages. These macrophages devour massive amounts of cholesteryl esters to become foam cells, a hallmark of the early stage of atherosclerotic lesions (3). Mechanistically, lipid metabolism disorder and chronic immuno-inflammation are the two well-recognized mechanism sunder lying foam cell formation.

Recently, mammalian target of rapamycin (mTOR) has drawn much attention as it plays a role in a variety of human diseases including AS, obesity, diabetes and cancer (4-7). The relationship between mTOR and AS is an emerging topic of research $(8,9)$. For instance, rapamycin, an mTOR inhibitor, has been shown to interfere with atherogenes is by attenuating inflammation and enhancing atherosclerotic plaque stability in various mammalian atherosclerotic models. Rapamycin, an mTOR inhibitor, mediates its effects without altering serum lipid levels, although it is currently used to treat hyperlipidemia and hypercholesterolemia $(10,11)$. The discrepant findings regarding rapamycin's effects on circulating lipid levels in animal models and human patients remain to be addressed. In addition, mTOR inhibition prevents lipid accumulation and 
upregulates cholesterol efflux $(12,13)$. For example, the mTOR signaling pathway was shown to be activated during THP-1 (Human acute monocytic leukemia cells) foam cell formation, and rapamycin or silencing mTOR suppressed this activation and increased the expression of cellular lipid efflux mediator adenosine triphosphate-binding cassette transporter A1 (ABCA1) (14). Collectively, these findings point to a potential correlation between mTOR and inflammation and cholesterol metabolism during foam cell formation in AS.

Recent studies also implicated mTOR in regulating diverse functions of professional antigen-presenting cells (APC), including dendritic cells (DCs) and macrophages (15). Consistently, rapamycin selectively promotes migration of mouse DCs to lymph nodes in vivo by enhancing the expression of C-C chemokine receptor type 7 (CCR7) (16), which is required for foam cell formation. CCR7 expression is mediated in part by liver X receptor (LXR) activation in atherosclerotic lesions, and both CCR7 and LXR are involved in plaque regression in $\mathrm{ApoE}^{-/}$mice $(17,18)$. Thus, both mTOR and LXR signaling mediate expression of CCR7 during plaque regression, suggesting a potential functional link between mTOR and LXR signaling.

The transcription factor nuclear factor- $\kappa \mathrm{B}(\mathrm{NF}-\kappa \mathrm{B})$ regulates various cytokines and chemical factors and inflammatory responses, which are predominant characteristics of AS development (19). Regulation of NF- $\mathrm{B}$ activity has been well studied, and one mechanism involves Sirtuin 1 (SIRT1). SIRT1 suppresses autophagy through activating NF-אB (20), but we revealed that SIRT1 can also prevent AS by activating LXR and inhibiting $\mathrm{NF}-\kappa \mathrm{B}$ signaling (21). Thus, SIRT1 appears to function upstream of the LXR/NF- $\kappa$ B axis. Whether SIRT1 mediates $\mathrm{NF}-\kappa \mathrm{B}$ in a positive or a negative way appears to be context-dependent.

Accumulating evidence indicates that there is a functional interaction between SIRT1 and mTOR. For example,rapamycin restored SIRT1-induced suppression of autophagy (20), and SIRT1 was required for the rapamycin-mediated effects on high glucose-induced mesangial cell senescence (22), suggesting a functional link between mTOR and SIRT1. Indeed, it was reported that SIRT1 negatively regulates mTOR and that mTOR inhibition increases SIRT1 activity $(23,24)$. These findings point to the possibility that mTOR and SIRT1 may be part of the same signaling pathway in AS pathogenesis, in which foam cell formation and egression are two important processes.

Herein, we hypothesized that mTOR signaling promotes monocyte-derived foam cell formation and inhibits foam cell egress through downregulating SIRT1/LXR/CCR7 and upregulating NF- $\kappa \mathrm{B}$ signaling. To test our hypothesis, we investigated the expression of key factors in mTOR and SIRT1/LXR/CCR7 signaling in U937-derived foam cells in vitro and discussed the functional relationship between the two signaling pathways.

\section{Materials and methods}

Reagents. Roswell Park Memorial Institute-1640 (RPMI-1640) medium was obtained from Gibco (Grand Island, NY, USA). Oil red $\mathrm{O}$ was purchased from Bio Basic Inc. (Markham, ON, Canada). 4'6-diamidino-2-phenylindole dihydrochloride
(DAPI) was purchased from Santa Cruz Biotechnology (Dallas, TX, USA). Oxidized low density lipoprotein (ox-LDL) was obtained from Yi Yuan Biotechnologies (Guangzhou, China). The following reagents were purchased from Sigma-Aldrich Corp. (St. Louis, MO, USA): sodium palmitate (PA), phorbol 12-myristate 13-acetate (PMA), rapamycin, lysophosphatidic acid (LPA), polyvinyl alcohol mounting medium with DABCO (PVA-DABCO; Sigma-Aldrich).

Antibodies. Monoclonal antibodies against mTOR and phosphorylated (p)-mTOR were purchased from Cell Signaling Technology (Danvers, MA, USA). p-ribosomal protein S6 kinase (p70S6K), SIRT1, LXR $\alpha$ and NF- $\mathrm{B}$ antibodies were purchased from Santa Cruz Biotechnology. Antibodies against CCR7 and tumor necrosis factor- $\alpha$ (TNF- $\alpha$ ) and donkey anti-rabbit IgG H\&L (Alexa Fluor ${ }^{\circledR}$ 594) were purchased from Abcam (Cambridge, UK). $\beta$-actin antibody was obtained from Zhon Shan Golden Brid (Beijing, China).

U937 cell differentiation and foam cell formation. The human monocytic cell line U937 was purchased from the Type Culture Collection (Chinese Academy of Sciences, Shanghai, China) and cultured in a humidified atmosphere at $37^{\circ} \mathrm{C}$ containing $5 \% \mathrm{CO}_{2}$ in RPMI-1640 supplemented with $10 \%$ fetal bovine serum (FBS; Biowest, Logan, UT, USA), penicillin (100 U/ml) and streptomycin $(100 \mathrm{mg} / \mathrm{ml})$. U937 cells were cultured in 6 -well plates $\left(2 \times 10^{6}\right.$ cells/well) and incubated with $160 \mathrm{nM}$ PMA for $24 \mathrm{~h}$, the differentiated macrophages were then harvested. Foam cell formation was induced by incubation of U937-derived macrophages with PA (0.2 mM) and ox-LDL $(80 \mu \mathrm{g} / \mathrm{ml})$ for another $24 \mathrm{~h}$.

Oil red $O$ staining. The U937-derived foam cells were induced successfully in 6-well cell culture clusters. Media was aspirated and the cells were fixed in $4 \%$ paraformaldehyde for half an hour. Subsequently, cells were stained with freshly diluted $0.3 \%$ Oil red O solution for $30 \mathrm{~min}$ at room temperature (RT). Thereafter, Oil red $\mathrm{O}$ solution was removed and cells were re-stained with hematoxylin for $10 \mathrm{sec}$ before mounting with glycerin gelatin. Cells were observed under a light microscope (Leica DM2500; Leica Microsystems, Mannheim, Germany) at x400 magnification and then photographed using a BX-51 camera (Olympus, Tokyo, Japan).

Cell viability and proliferation assay. U937 cell viability was measured by 3-(4,5-dimethylthiazol-2-yl)-2,5-diphenyltetrazolium bromide (MTT) assay. Briefly, cells were cultured in 96-well plates $\left(1 \times 10^{4}\right.$ cells/well) with $100 \mu \mathrm{l}$ medium. The medium was refreshed with different concentrations of rapamycin (0.01-100 $\mathrm{nM}$ ) and cultured for 24 h.Thereafter, MTT $(5 \mathrm{mg} / \mathrm{ml}$ ) was added to each well including control (one set of wells with MTT but no cells), and the cells were cultured for another $4 \mathrm{~h}$ at $37^{\circ} \mathrm{C}$ Triple liquid [10\% sodium dodecyl sulfate (SDS), $5 \%$ isobutanol, $0.012 \mathrm{~mol} / 1$ hydrochloric acid, dissolved in distilled water] was added to each well. After covering with tinfoil and agitating cells on an orbital shaker for $15 \mathrm{~min}$, the absorbance of each sample was measured at $570 \mathrm{~nm}$ by a microtiter plate reader (Multiskan MK3; Thermo Scientific, Helsinki, Finland). All experiments were repeated in triplicate. 
Table I. Primer sequences used for quantitative real-time PCR.

\begin{tabular}{lll}
\hline Gene & Forward sequences $\left(5^{\prime}\right.$ to $\left.3^{\prime}\right)$ & Reverse sequences $\left(5^{\prime}\right.$ to $\left.3^{\prime}\right)$ \\
\hline mTOR & CATCATGTTGCGGATGGCTC & AGCATCAGGTTGGATGGGTG \\
p70S6K & ACTAGTGTGAACAGAGGGCC & CATTGCCTTTCCATAGCCCC \\
SIRT1 & GCTCGCCTTGCTGTAGACTTCC & GCAACCTGTTCCAGCGTGTCT \\
NF-kB & TGTCCAGCTTCGGAGGAAAT & TCTGACGTTTCCTCTGCACT \\
TNF- $\alpha$ & TCTTCTGCCTGCTGCACTTT & TCAGCTTGAGGGTTTGCTACA \\
LXR- $\alpha$ & ATGCCGAGTTTGCCTTGC & CATCCGTGGGAACATCAGT \\
CCR7 & CATCAGCATTGACCGCTACG & GTATCCAGATGCCCACACAGG \\
ABCA1 & TCCTCCTGGTGAGTGCTTTG & GGGACTCCTCTCAAAAGGGC \\
GAPDH & TGAACGGGAAGCTCACTGG & TCCACCACCCTGTTGCTGTA
\end{tabular}

mTOR, mammalian target of rapamycin; p70S6K, ribosomal protein S6 kinase; TNF- $\alpha$, tumor necrosis factor $\alpha$; CCR7, C-C chemokine receptor type 7; SIRT1, sirtuin 1; LXR- $\alpha$, liver X receptor $\alpha$; ABCA1, ATP-binding cassette transporter A1; GAPDH, glyceraldehyde 3-phosphate dehydrogenase.

Immunofluorescence staining. For immunofluorescence analysis, U937-derived foam cells were fixed in $4 \%$ paraformaldehyde in 1X phosphate-buffered saline (PBS) for $15 \mathrm{~min}$ and permeabilized with $0.1 \%$ Triton X-100 in $1 \mathrm{X}$ PBS for 10 min. After being blocked with $3 \%$ bovine serum albumin (BSA) in 1X PBS for 30 min, cells were incubated with $\mathrm{NF}-\kappa \mathrm{B}$ p65 antibody at $4{ }^{\circ} \mathrm{C}$ overnight and washed twice with $0.1 \%$ Tween-20 in $1 \mathrm{X}$ PBS every $5 \mathrm{~min}$, followed by incubation for $1 \mathrm{~h}$ with Alexa 594-labeled anti-rabbit IgG secondary antibody at RT. Cell nuclei were stained with $200 \mathrm{ng} / \mathrm{ml}$ DAPI for $10 \mathrm{~min}$ at RT and the cells were mounted in PVA-DABCO. Images were captured using a FV1000 confocal laser scanning microscope (Olympus).

Real-time reverse transcription quantitative PCR (real-time $R T-P C R$ ) analysis. Total RNA was extracted from cells using TRIzol reagent RNAiso Plus (Takara, Dalian, China) according to the manufacturer's instructions. The concentration and purity of the RNA were measured and determined by NanoDrop 2000c (Thermo Scientific, Waltham, MA, USA) and the OD260/280 value was 1.9-2.1. Extracted RNA was reverse-transcribed with PrimeScript ${ }^{\mathrm{TM}}$ RT reagent kit with gDNA eraser (Takara). Real-time PCR was performed using ABI Prism 7500 systems (Applied Biosystems, Foster City, CA, USA) with GoTaq ${ }^{\circledR}$ qPCR Master Mix (Promega Corporation, Madison, WI, USA). Amplification was carried out in a total volume of $20 \mu \mathrm{l}$ and 40 cycles after pre-denaturation $\left(95^{\circ} \mathrm{C}\right.$ for $10 \mathrm{~min}$ ) followed by: $95^{\circ} \mathrm{C}$ for $15 \mathrm{sec}$ and $60^{\circ} \mathrm{C}$ for $1 \mathrm{~min}$. The primer sequences used in real-time-PCR are shown in Table I. Experiments were performed in triplicate for each sample. The relative amount of mRNA was calculated using the comparative CT method. Glyceraldehyde 3-phosphate dehydrogenase (GAPDH) served as the reference housekeeping gene. A melting curve analysis was performed after amplification to verify the accuracy of the amplicon.

Western blot analysis. Cells were lysed using RIPA lysis buffer containing $150 \mathrm{mM} \mathrm{NaCl}, 1.0 \% \mathrm{NP}-40,50 \mathrm{mM}$ Tris $\cdot \mathrm{HCl}$ ( $\mathrm{pH} 7.4), 0.5 \%$ sodium deoxycholate, and $0.1 \%$ SDS. Proteins were separated by $10 \%$ SDS-polyacrylamide gel electrophoresis (SDS-PAGE) and transferred onto nitrocellulose membranes (Merck Millipore, Darmstadt, Germany). After being washed once with Tris-buffered saline (TBS), the membranes were blocked in 5\% skimmed milk in TBS-Tween (TBST) for $1 \mathrm{~h}$ and incubated with primary antibodies at $4^{\circ} \mathrm{C}$ overnight, followed by another incubation with an appropriate secondary antibody at RT for $1 \mathrm{~h}$. The immunoblots were visualized by enhanced chemiluminescence (Advansta, Menlo Park, CA, USA). Signal intensities were measured by Quantity One software (Bio-Rad, Hercules, CA, USA).

Statistics. Data are presented as means \pm standard deviation (SD) from at least three independent experiments. Results were statistically analyzed with unpaired Student's t-test or one-way analysis of variance (ANOVA) using the statistical software GraphPad Prism 5.01 (La Jolla, CA, USA). In all tests, $\mathrm{P}<0.05$ was considered statistically significant.

\section{Results}

$P A$ and ox-LDL treatment induces lipid droplet accumulation and upregulates $p$-mTOR and p-p70S6K during foam cell formation. Foam cell formation was induced in U937-derived macrophages by PA and ox-LDL treatment. Following macrophage differentiation induced by PMA for $24 \mathrm{~h}$, the U937-derived macrophages were divided into three groups: the control group, the PA group, and the PA+ ox-LDL group. After incubation for another $24 \mathrm{~h}$, a larger number of lipid droplets accumulated in the cells in the PA+ ox-LDL group compared to the control and PA groups, as revealed by Oil red O staining (Fig. 1A-C). We also evaluated changes in p-mTOR and p-p70S6K protein levels in cells from these three groups by Western blot analysis. As demonstrated in Fig. 1D and E, p-mTOR and p-p70S6K expression were upregulated in the PA+ ox-LDL group compared to the other two groups. Thus, foam cell formation upregulates expression of $\mathrm{p}$-mTOR and p-p70S6K.

LPA upregulates $p$-mTOR and p-p70S6K but decreases SIRT1 expression. LPA has been reported to promote atherosclerotic 
A

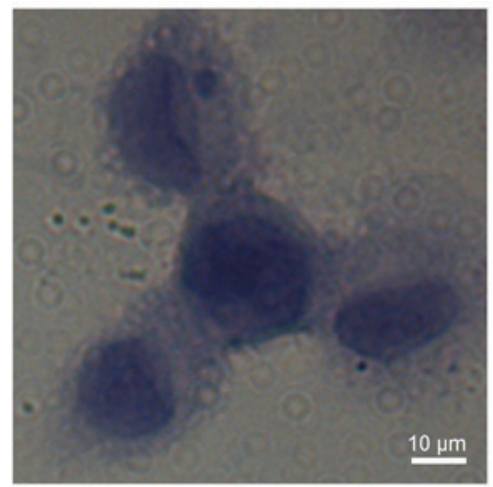

D

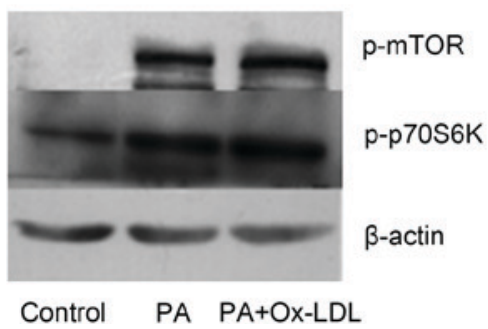

B

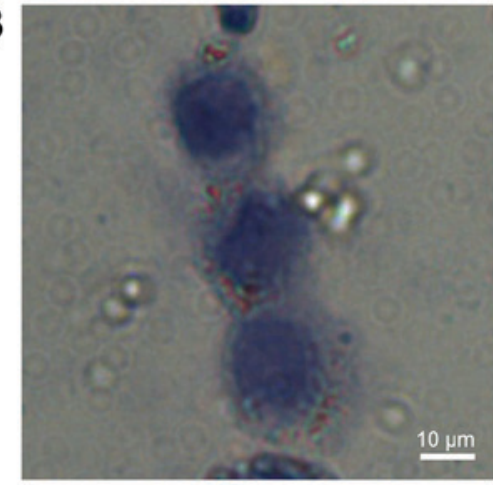

$E$

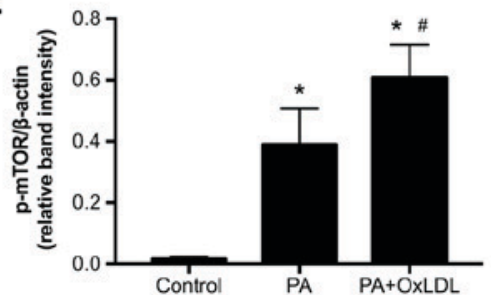

C
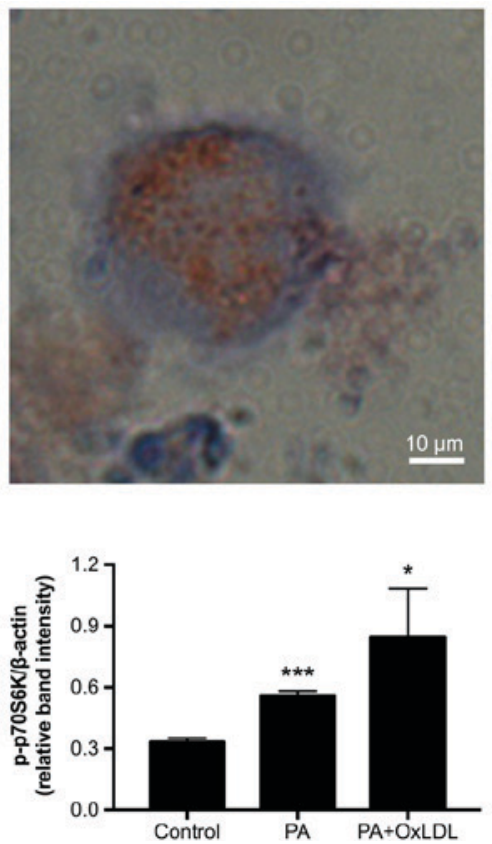

Figure 1. PA and ox-LDL treatment induces lipid droplet accumulation and upregulate p-mTOR and p-p70S6K during foam cell formation. (A-C) Oil red O staining of U937 cells in the control group (A), the PA $(0.2 \mathrm{mM})$ group (B), and the PA $(0.2 \mathrm{mM})+$ ox-LDL $(80 \mu \mathrm{g} / \mathrm{ml})$ group $(\mathrm{C})$. (D and E) p-mTOR and p-p70S6K were upregulated during foam cell formation as analyzed by western blot analysis (D). E shows the quantitative analysis of data in $\mathrm{D}$. "P<0.05 vs. control; ${ }^{\#} \mathrm{P}<0.05$ vs. $\mathrm{PA} ;{ }^{* * *} \mathrm{P}<0.001$ vs. control; ${ }^{*} \mathrm{P}<0.05$ vs. control. Data are presented as mean $\pm \mathrm{SD}$ from three independent experiments.
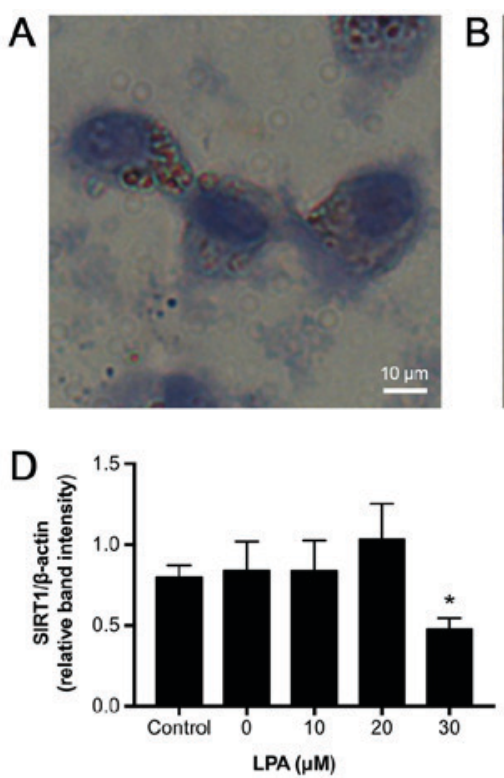
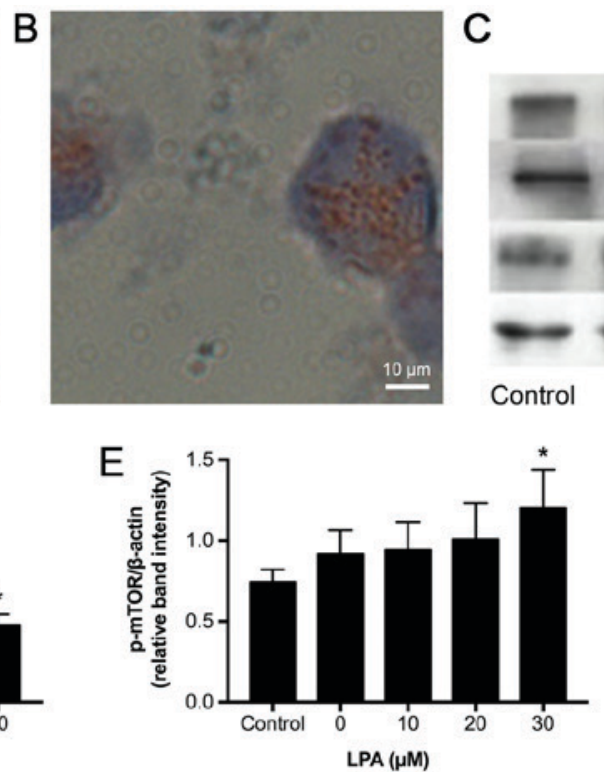

C
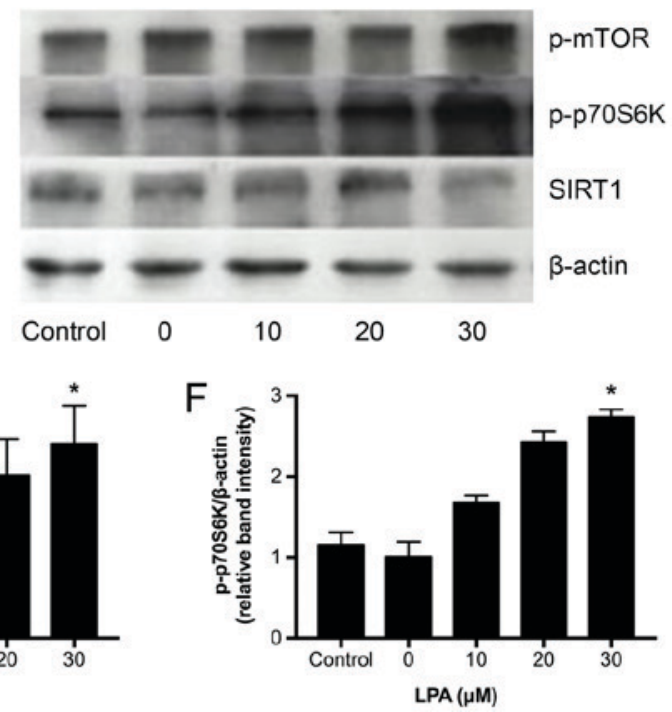

Figure 2. LPA upregulates p-mTOR and p-p70S6K expression but decreases SIRT1 expression. Oil red O staining of U937 foam cells in untreated cells (A) and treated cells (B) with LPA. p-mTOR, p-p70S6K and SIRT1 expression in foam cells treated with different concentrations of LPA was evaluated by western blot analysis (C). (D-F) show the quantitative analysis of p-mTOR, p-pS6K and SIRT1. ${ }^{*} \mathrm{P}<0.05$ vs. control. Data are expressed as mean \pm SD from at least three independent experiments.

lesion formation by promoting monocyte migration from the bloodstream into the vascular wall and decreasing monocyte-derived cell emigration from the vessel wall (25).To better understand the effects of atherogenetic conditions on mTOR signaling, U937-derived macrophages were incubated with different LPA concentrations $(0,10,20$ and $30 \mu \mathrm{M})$ to induce foam cell formation. We used Oil red $\mathrm{O}$ staining to observe lipid accumulation and found that $30 \mu \mathrm{M}$ LPS increased the number of lipid droplets in the cultured cells (Fig. 2A and B).
Next, the effects of LPA on the expression of p-mTOR, p-p70S6K and SIRT1 were assessed by western blot analysis. As shown in Fig. 2E and F, p-mTOR and its downstream factor p-p70S6K were upregulated by $30 \mu \mathrm{M}$ LPA, while SIRT1 expression was downregulated. Therefore, $30 \mu \mathrm{M}$ LPA was chosen for the subsequent foam cell formation experiments.

Rapamycin reduces cell viability. To determine whether rapamycin affects cell viability, U937 cells were incubated 


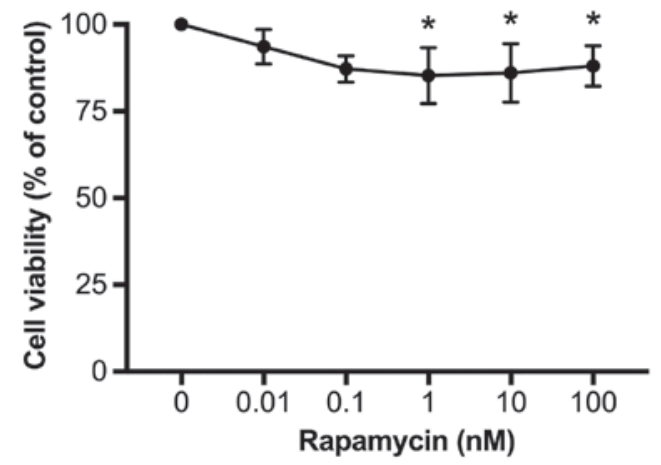

Figure 3. Rapamycin reduces cell viability. U937 cells were exposed to different concentrations (as indicated) of rapamycin for $24 \mathrm{~h}$ and cell viability was determined by MTT analysis. Data are presented as mean \pm SD from three independent experiments." $\mathrm{P}<0.05$ vs. control (0).

with rapamycin at different concentrations $(0,0.01,0.1,1,10$, and $100 \mathrm{nM}$ ) in RPMI-1640 for $24 \mathrm{~h}$ and then cell viability was evaluated by the MTT method. As demonstrated in Fig. 3, rapamycin treatment dose-dependently decreased cell viability, from $94 \pm 2 \%$ at $0.01 \mathrm{nM}$ to $85 \pm 4 \%$ at $1 \mathrm{nM}$ $(\mathrm{P}<0.05$, vs. 0$)$. Increases in rapamycin concentration to 10 or $100 \mathrm{nM}$ did not further reduce cell viability $(86 \pm 4$ and $88 \pm 2 \%$, respectively). Therefore, in order to achieve the maximal inhibitory effects, $100 \mathrm{nM}$ rapamycin was used for the subsequent experiments.

Rapamycin reduces lipid droplet accumulation and inhibits mTOR signaling in ox-LDL, PA and LPA induced foam cells. We next investigated if rapamycin could inhibit mTOR in U937-derived foam cells induced by PA, ox-LDL and LPA. As expected, Oil red $\mathrm{O}$ staining revealed accumulation of lipid droplets in the foam cells treated with PA, ox-LDL and LPA, which was significantly decreased by $100 \mathrm{nM}$ rapamycin (Fig. 4A and $\mathrm{B}$ ). The effects of rapamycin on mRNA expression of mTOR and p70S6K were also assessed. As shown in Fig. 4C and D, exposure to $100 \mathrm{nM}$ rapamycin at varying time-points $(6,12$, and $24 \mathrm{~h})$ decreased mTOR and p70S6K expression compared to control $(0 \mathrm{~h})$, with the most significant inhibitory effect observed at $12 \mathrm{~h}$. Consistent with the above observations, western blot analysis revealed that $12 \mathrm{~h}$ rapamycin treatment substantially reduced mTOR, p-mTOR, and p-p70S6K $(\mathrm{P}<0.05)$ protein expression $($ Fig. 4E-H). Based on these observations, we conclude that rapamycin suppresses mTOR signaling, and we used a $12 \mathrm{~h}$ treatment in the subsequent experiments.

Rapamycin increases SIRT1 signaling but decreases $N F-\kappa B$ activity in foam cells. Next, we investigated the functional relationship between mTOR and SIRT1 signaling. To do so we measured the expression of SIRT1 signaling factors, including SIRT1, LXR $\alpha$, CCR7, ABCA1 and NF- $\kappa$ B, in the foam cells in the absence or presence of rapamycin using real-time RT-PCR and Western blot analyses. SIRT1, LXR $\alpha$, CCR7 and ABCA1 mRNA levels were significantly decreased but NF- $\kappa$ B and its downstream factor TNF- $\alpha$ was upregulated in foam cells. However, rapamycin $(100 \mathrm{nM})$ treatment for $12 \mathrm{~h}$ significantly increased SIRT1, LXR $\alpha$, CCR7 and ABCA1 mRNA levels but decreased the NF- $\kappa \mathrm{B}$ and TNF- $\alpha$ (Fig. 5A-F). In line with the above observations, SIRT1, LXR $\alpha$ and CCR7 protein expression were decreased but $N F-\kappa B$ and TNF- $\alpha$ were increased in foam cells, and these changes were reversed by $12 \mathrm{~h}$ rapamycin treatment (Fig. 5G-L). Immunofluorescence analysis showed that foam cells exhibited more $\mathrm{NF}-\kappa \mathrm{B}$ nuclear staining compared to the control cells, suggesting that $N F-\kappa B$ signaling is activated in foam cells. However, this increased
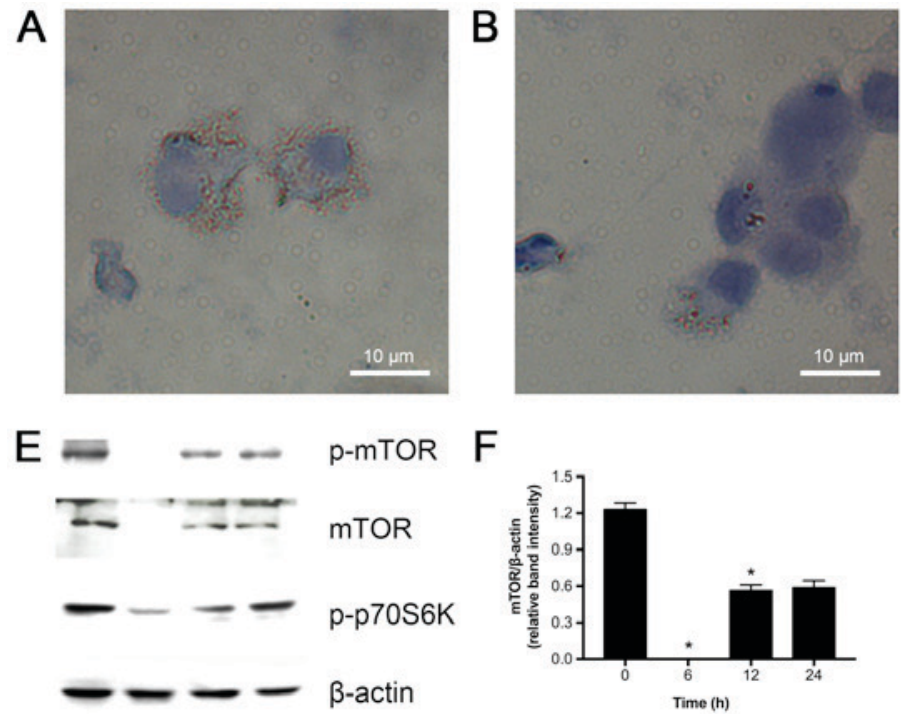

$\mathrm{F}$

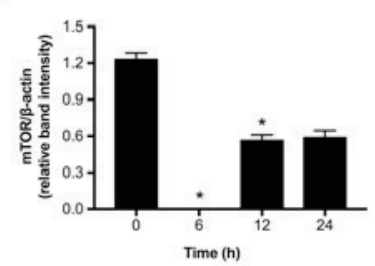

C

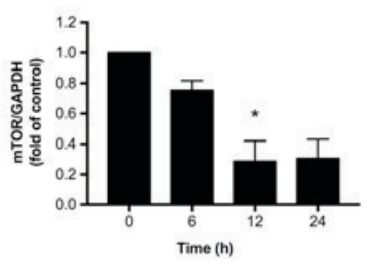

G

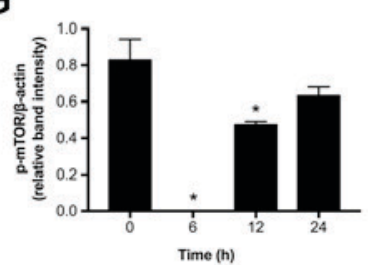

D
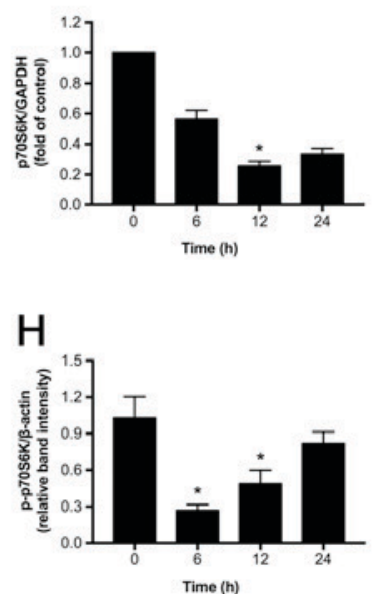

Figure 4. Rapamycin reduces lipid droplets and inhibits mTOR signaling in foam cells induced by ox-LDL, PA and LPA. Oil red O staining of U937-derived foam cells with rapamycin (A) or without rapamycin (B). The effect of rapamycin on the expression of mTOR and p70S6K during foam cell formation induced by ox-LDL, PA and LPA. (C and D) mRNA expression of mTOR and p70S6K at different time points $(0,6,12$, and $24 \mathrm{~h})$ of incubation with rapamycin in foam cells was assessed by real-time RT-PCR. * $\mathrm{P}<0.05$ vs. control. (E) Protein levels of mTOR and p-mTOR and p-p70S6K at different time-points $(0,12,24$, and $48 \mathrm{~h}$ ) of incubation with rapamycin were assessed by western blot analysis. $\beta$-actin was used as a loading control. (E-H) Quantitative analysis of E by Quantity One software. ${ }^{*} \mathrm{P}<0.05$ vs. control. Data are expressed as mean $\pm \mathrm{SD}$ from three independent experiments. 
A

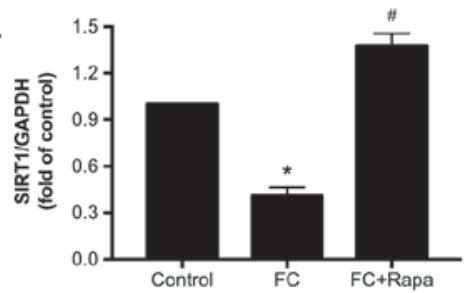

D

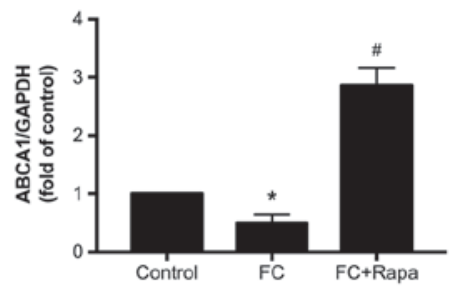

G

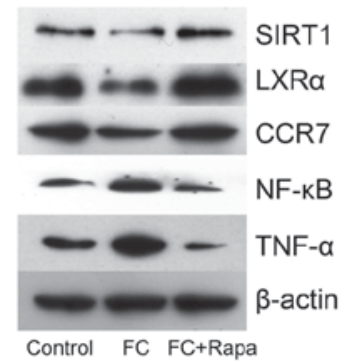

$J$

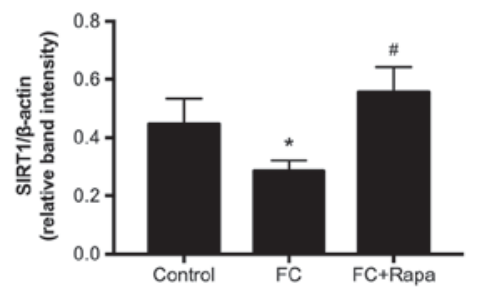

B

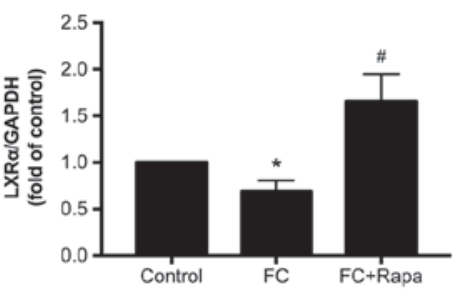

E

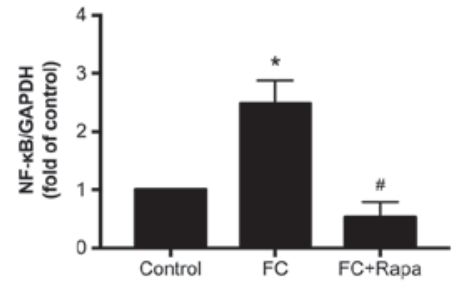

$\mathrm{H}$

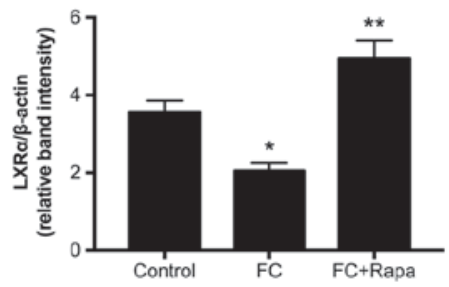

$\mathrm{K}$

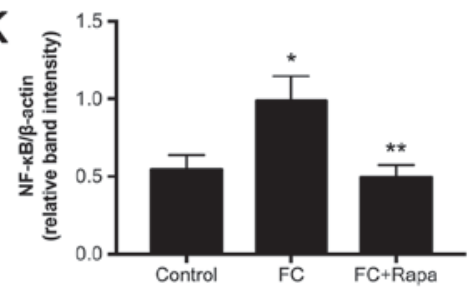

C

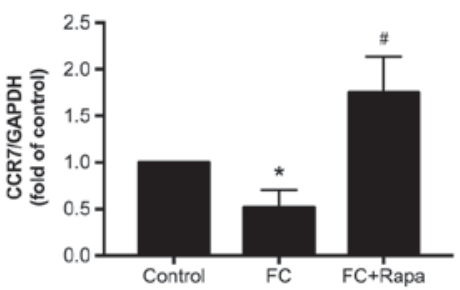

$\mathrm{F}$

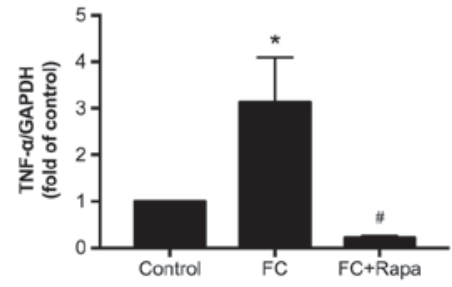

1

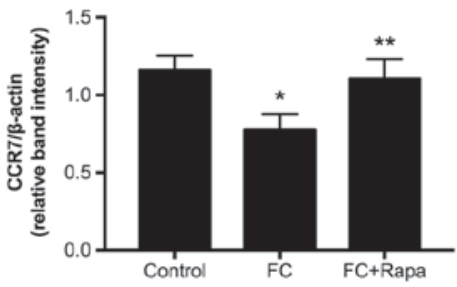

L

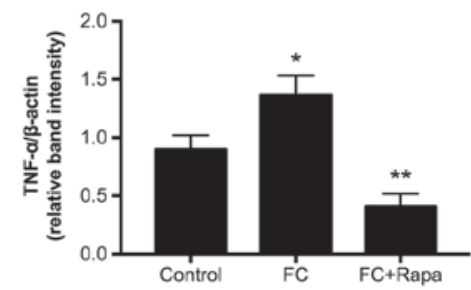

Figure 5. Rapamycin increases SIRT1 signaling but decreases NF- $\kappa$ B activity in foam cells. (A-F) mRNA expression of each indicated gene was assessed by real-time RT-PCR. (G-L) Protein expression of each indicated gene was assessed by western blot analysis. $\beta$-actin was used as a loading control. ${ }^{*} \mathrm{P}<0.05$ vs. control. Data are represented as mean \pm SD from triplicates for RT-PCR and from three independent experiments for western blot analysis. Rapa, rapamycin; FC, foam cells.

nuclear expression of $\mathrm{NF}-\kappa \mathrm{B}$ was decreased by rapamycin treatment (Fig. 6). Thus, our data indicate that rapamycin increases SIRT1 signaling but decreases NF- $\kappa$ B activity in foam cells.

\section{Discussion}

In the present study, we established a cell model of foam cell formation, which is the main pathological process in early AS. When U937 cells were cultured in the presence of high fat and high cholesterol, mTOR and p70S6K expression, as well as their phosphorylated forms, were upregulated indicating that mTOR signaling was activated during foam cell formation. These findings are consistent with accumulating evidence showing that mTOR signaling plays an imperative role in lipid metabolism (26) and AS (27). In our in vitro model, we further explored the functional link between mTOR and SIRT1 signaling during the formation of foam cells and concluded that mTOR promotes foam cell formation through suppressing SIRT1 signaling.
Recent studies supported the notion that mTOR signaling contributes to AS pathogenesis and that mTOR inhibitors, such as rapamycin, have pleiotropic anti-atherosclerotic effects that prevent or delay AS progression (8). In the present study, we used U937 cells to establish a cell model of foam cell formation induced by PA + ox-LDL, and we also measured the expression levels of mTOR and SIRT1in foam cells treated with LPA, which mimics atherosclerotic lesions in vivo. LPA, as a lipid mediator, has been reported to play important roles in inflammation and AS $(28,29)$. Consistent with the above reports, we observed increased levels of mTOR and its downstream p70S6K in foam cells, suggesting that mTOR signaling is activated during foam cell formation. As expected, these increases were attenuated by $100 \mathrm{nM}$ rapamycin, with a maximum inhibition achieved at $12 \mathrm{~h}$ in PA + ox-LDL-induced foam cells. Intriguingly, the increase in mTOR activity was coincidental with the decrease in SIRT1 expression, and rapamycin also suppressed the decrease in SIRT1. Similarly, in the LPA induced atherosclerotic lesion model, mTOR signaling was 

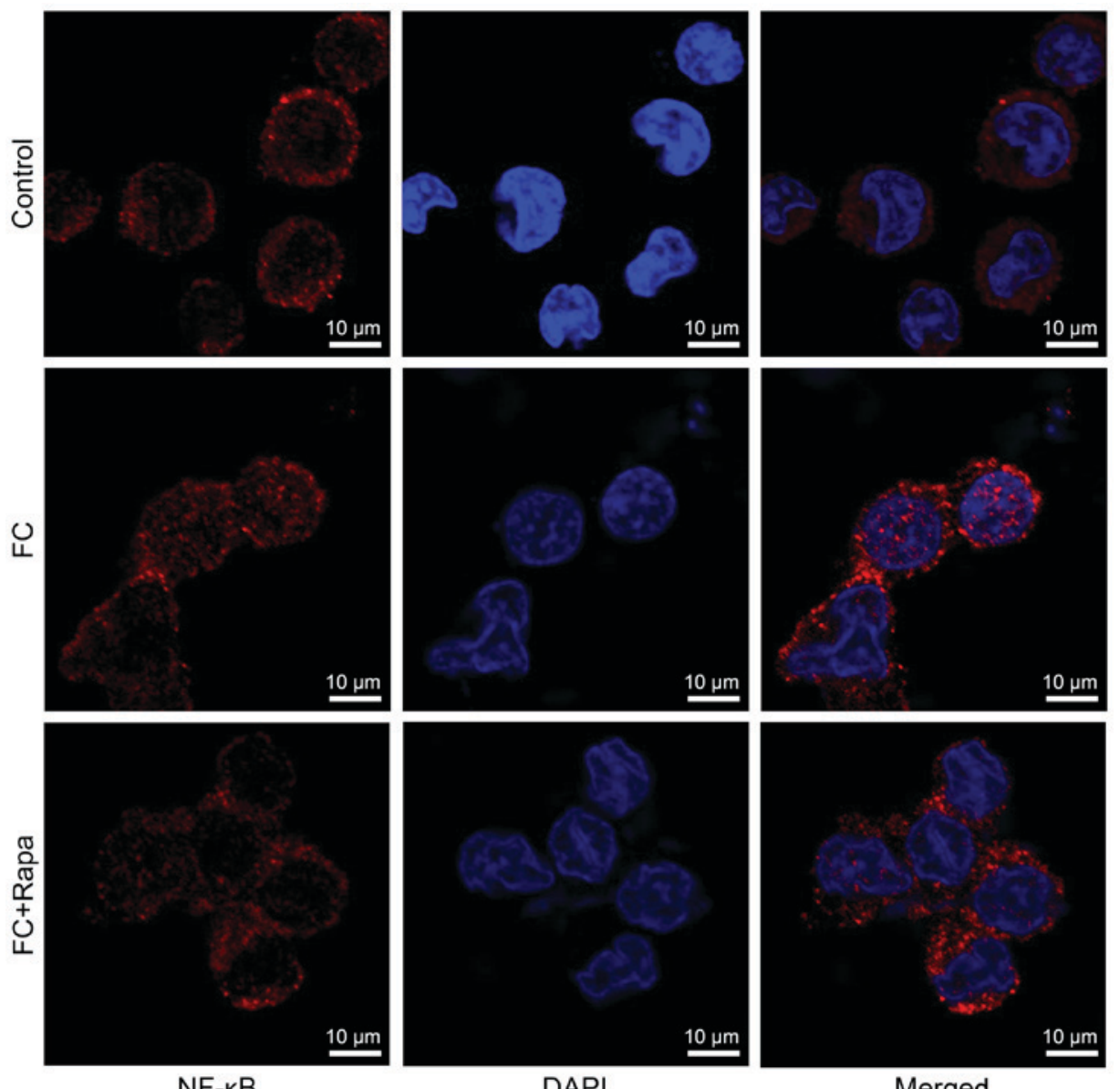

NF-KB

DAPI

Merged

Figure 6. Rapamycin reduces nuclear NF-кB expression in foam cells. Immunofluorescence staining was performed in control (U973 cells), foam cells (FC) and FC treated with $100 \mathrm{nM}$ rapamycin (FC+Rapa). Red, NF-кB; blue, DAPI as a nuclear counterstain. Images were captured at a x1200 magnification. The images are representative of three independent experiments that showed similar results.

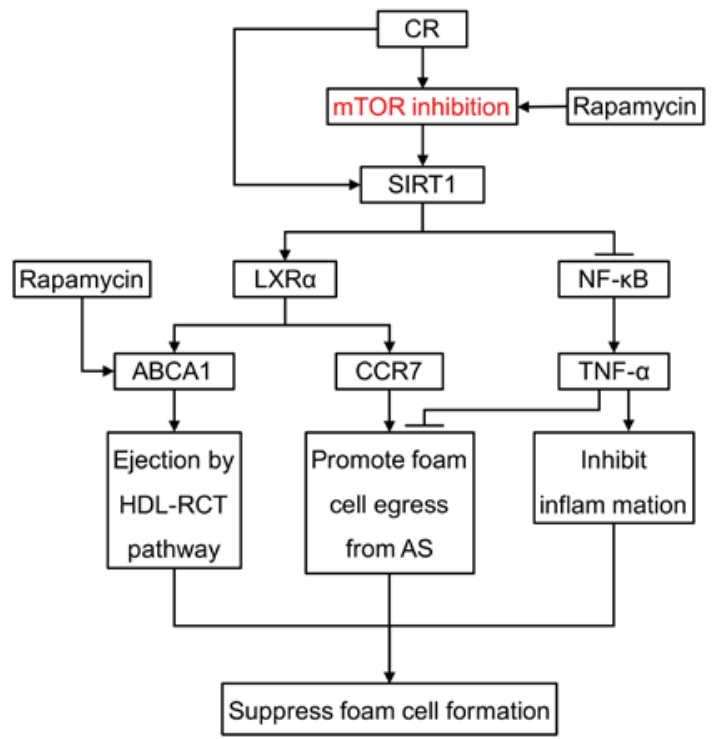

Figure 7. A model of mTOR signaling pathways involved in AS. Solid line with arrows represents activation of gene expression; lines without arrows indicate suppression of gene expression.

significantly increased but SIRT1 was decreased. Previous findings revealed that SIRT1 negatively mediated mTOR under stress conditions (30), and that SIRT1 negatively regulated mTOR and vice versa $(23,24)$. Our findings further support the potential link between elevated mTOR signaling and decreased SIRT1 activity in foam cells, although the evidence for a direct functional correlation between these two remains unknown.

Normal mTOR activity in endothelial cells requires proper cholesterol trafficking (31). LXR $\alpha$ and its downstream factors, CCR7 and ABCA1, contribute to cholesterol trafficking, and thus are involved in AS regression. For instance, increased expression of CCR7, as a requisite factor for DC migration, was shown to play a crucial role in AS regression in ApoE//mice (17). Also, LXR-CCR7 signaling participated in monocyte-derived cell egression during AS regression in mice (18). Therefore, activation of the LXR $\alpha$-CCR7-ABCAlaxis is believed to mitigate AS. Indeed, in the present study, LXR $\alpha$ expression, and its downstream factors CCR7 and ABCA1, were reduced during foam cell formation. However, rapamycin treatment rescued this reduction. Combined with our previous findings suggesting that SIRT1 prevents AS through LXR $\alpha$ signaling (21), it is possible that mTOR inhibition may retard AS pathogenesis and promote foam cell egression by upregulating SIRT1, further enhancing the LXR $\alpha$-CCR7 signaling pathway. Therefore, we believe that the SIRT1-LXR $\alpha$-CCR7 signaling pathway mediates rapamycin-induced beneficial effects on AS. 
AS is also regarded as a low level chronic immumoinflammatory disease. NF- $\mathrm{BB}$, a core transcription factor involved in the pro-inflammatory response, controls expression of several important genes (including TNF- $\alpha$ ) directing the initiation and progression of AS (19). Activated endothelial $\mathrm{NF}-\kappa \mathrm{B}$ signaling promotes macrophage recruitment to atherosclerotic plaques (32). More specifically, macrophage-derived foam cells can secrete TNF- $\alpha$ and other cytokines to recruit immune cells, contributing to the formation of atherosclerotic plaques, further increasing the risk of cardiovascular events (19). Also, inflammation downregulated the expression of ABCA1 and LXR $\alpha$, which may reduce cholesterol efflux (33). However, SIRT1 inhibits the transcriptional activity of NF- $\mathrm{KB}$ via deacetylation (34), and regulates the efficiency of NF- $\kappa B$ signaling via altering pro-inflammatory responses (35). Consistent with the above observations, our study showed that NF- $\kappa \mathrm{B}$ expression and its target gene, TNF- $\alpha$, were enhanced during foam cell formation, but this effect was reversed by rapamycin, indicating that $\mathrm{mTOR}$ inhibition may suppress foam cell formation by enhancing SIRT1-NF- $\mathrm{kB}$ signaling.

Calorie restriction (CR), which limits calorie intake without compromising essential nutrients, could extend lifespan and delay age-related diseases, such as AS, by increasing SIRT1 activity (36). Our previous study showed that SIRT1 expression was increased under CR (21). In addition, it was shown that CR slowed aging and delayed age-associated diseases by deactivating the mTOR pathway $(37,38)$, indicating that both SIRT1 and mTOR signaling are involved in CR-linked responses. Similarly, in the present study we found that SIRT1 was activated by mTOR inhibition in foam cells, followed by enhanced expression of LXR and CCR7 and suppressed NF- $\kappa B$ signaling. Thus, it appears that the functional interaction between mTOR and SIRT1 represents a general paradigm that is involved in different pathophysiological settings. We speculate that mTOR inhibition may selectively increase SIRT1-LXR-CCR7 and decrease NF- $\mathrm{kB}$ signaling, thus repressing foam cell formation and promoting foam cell egression (Fig. 7).

In conclusion, in the present study we demonstrated that mTOR signaling is upregulated during foam cell formation induced by PA and ox-LDL, as well as LPA-mediated AS that mimics a high fat and high cholesterol environment. Furthermore, we showed that potentiated mTOR signaling is coincident with enhanced NF- $\mathrm{KB}$ and decreased SIRT1-LXR $\alpha$-CCR7 signaling. The changes in the above-mentioned signaling pathways were reversed by the mTOR inhibitor, rapamycin. Hence, mTOR signaling may promote foam cell formation and inhibit foam cell egression through suppressing SIRT1-LXR-CCR7 and enhancing NF- $\mathrm{BB}$ signaling, while mTOR inhibition or upregulation of SIRT1 signaling may promote foam cell egress and reduce atherosclerotic plaques clinically.

\section{Acknowledgements}

The work was supported by grants from the Natural Science Foundation of China (no. 81270382), the Medical Scientific Research Foundation of Guangdong Province (no. A2014445) and the Research Fund for the Doctoral Program of Higher Education (no. 20134402110004).

\section{References}

1. Yusuf S, Reddy S, Ounpuu S and Anand S: Global burden of cardiovascular diseases: Part I: General considerations, the epidemiologic transition, risk factors, and impact of urbanization. Circulation 104: 2746-2753, 2001.

2. Go AS, Mozaffarian D, Roger VL, Benjamin EJ, Berry JD, Blaha MJ, Dai S, Ford ES, Fox CS, Franco S, et al: Heart disease and stroke statistics-2014 update: A report from the American Heart Association. Circulation 129: e28-e292, 2014.

3. Falk E: Pathogenesis of atherosclerosis. J Am Coll Cardiol 47 (8 Suppl): C7-C12, 2006.

4. Cruzado JM: Nonimmunosuppressive effects of mammalian target of rapamycin inhibitors. Transplant Rev (Orlando) 22: 73-81, 2008.

5. Yang Z and Ming XF: mTOR signalling: The molecular interface connecting metabolic stress, aging and cardiovascular diseases. Obes Rev 13 (Suppl 2): S58-S68, 2012.

6. Yang Q and Guan KL: Expanding mTOR signaling. Cell Res 17: 666-681, 2007.

7. Sciarretta S, Volpe M and Sadoshima J: Mammalian target of rapamycin signaling in cardiac physiology and disease. Circ Res 114: 549-564, 2014.

8. Martinet W, De Loof H and De Meyer GR: mTOR inhibition: A promising strategy for stabilization of atherosclerotic plaques. Atherosclerosis 233: 601-607, 2014.

9. Johnson SC, Rabinovitch PS and Kaeberlein M: mTOR is a key modulator of ageing and age-related disease. Nature 493: 338-345, 2013.

10. Mueller MA, Beutner F, Teupser D, Ceglarek U and Thiery J: Prevention of atherosclerosis by the mTOR inhibitor everolimus in $\mathrm{LDLR}^{-1-}$ mice despite severe hypercholesterolemia. Atherosclerosis 198: 39-48, 2008.

11. Chen WQ, Zhong L, Zhang L, Ji XP, Zhang M, Zhao YX, Zhang $\mathrm{C}$ and Zhang $\mathrm{Y}$ : Oral rapamycin attenuates inflammation and enhances stability of atherosclerotic plaques in rabbits independent of serum lipid levels. Br J Pharmacol 156: 941-951, 2009.

12. Ma KL, Ruan XZ, Powis SH, Moorhead JF and Varghese Z: Anti-atherosclerotic effects of sirolimus on human vascular smooth muscle cells. Am J Physiol Heart Circ Physiol 292: H2721-H2728, 2007.

13. Mathis AS, Jin S, Friedman GS, Peng F, Carl SM and Knipp GT: The pharmacodynamic effects of sirolimus and sirolimus-calcineurin inhibitor combinations on macrophage scavenger and nuclear hormone receptors. J Pharm Sci 96: 209-222, 2007

14. Yu M, Kang X, Xue $\mathrm{H}$ and Yin H: Toll-like receptor 4 is up-regulated by mTOR activation during THP-1 macrophage foam cells formation. Acta Biochim Biophys Sin (Shanghai) 43: 940-947, 2011.

15. Thomson AW, Turnquist HR and Raimondi G: Immunoregulatory functions of mTOR inhibition. Nat Rev Immunol 9: 324-337, 2009.

16. Sordi V, Bianchi G, Buracchi C, Mercalli A, Marchesi F, D'Amico G, Yang CH, Luini W, Vecchi A, Mantovani A, et al: Differential effects of immunosuppressive drugs on chemokine receptor CCR7 in human monocyte-derived dendritic cells: Selective upregulation by rapamycin. Transplantation 82: 826-834, 2006.

17. Trogan E, Feig JE, Dogan S, Rothblat GH, Angeli V, Tacke F, Randolph GJ and Fisher EA: Gene expression changes in foam cells and the role of chemokine receptor CCR7 during atherosclerosis regression in ApoE-deficient mice. Proc Natl Acad Sci USA 103: 3781-3786, 2006.

18. Feig JE, Pineda-Torra I, Sanson M, Bradley MN, Vengrenyuk Y, Bogunovic D, Gautier EL, Rubinstein D, Hong C, Liu J, et al: LXR promotes the maximal egress of monocyte-derived cells from mouse aortic plaques during atherosclerosis regression. J Clin Invest 120: 4415-4424, 2010.

19. Baker RG, Hayden MS and Ghosh S: NF-кB, inflammation, and metabolic disease. Cell Metab 13: 11-22, 2011.

20. Takeda-Watanabe A, Kitada M, Kanasaki K and Koya D: SIRT1 inactivation induces inflammation through the dysregulation of autophagy in human THP-1 cells. Biochem Biophys Res Commun 427: 191-196, 2012.

21. Zeng HT, Fu YC, Yu W, Lin JM, Zhou L, Liu L and Wang W: SIRT1 prevents atherosclerosis via liver-X-receptor and NF- $\mathrm{B}$ signaling in a U937 cell model. Mol Med Rep 8: 23-28, 2013. 
22. Zhang S, Cai G, Fu B, Feng Z, Ding R, Bai X, Liu W, Zhuo L, Sun L, Liu F and Chen X: SIRT1 is required for the effects of rapamycin on high glucose-inducing mesangial cells senescence. Mech Ageing Dev 133: 387-400, 2012.

23. Medvedik O, Lamming DW, Kim KD and Sinclair DA: MSN2 and MSN4 link calorie restriction and TOR to sirtuin-mediated lifespan extension in saccharomyces cerevisiae. PLoS Biol 5: e261, 2007.

24. Ghosh HS, McBurney M and Robbins PD: SIRT1 negatively regulates the mammalian target of rapamycin. PLoS One 5: e9199, 2010.

25. Cui MZ: Lysophosphatidic acid effects on atherosclerosis and thrombosis. Clin Lipidol 6: 413-426, 2011.

26. Lamming DW and Sabatini DM: A Central role for mTOR in lipid homeostasis. Cell Metab 18: 465-469, 2013.

27. Ma KL, Liu J, Wang CX, Ni J, Zhang Y, Wu Y, Lv LL, Ruan XZ and Liu BC: Activation of mTOR modulates SREBP-2 to induce foam cell formation through increased retinoblastoma protein phosphorylation. Cardiovasc Res 100: 450-460, 2013.

28. Fueller M, Wang DA, Tigyi G and Siess W: Activation of human monocytic cells by lysophosphatidic acid and sphingosine-1-phosphate. Cell Signal 15: 367-375, 2003.

29. Bot M, Bot I,Lopez-Vales R, van de Lest CH, Saulnier-Blache JS Helms JB, David S, van Berkel TJ and Biessen EA: Atherosclerotic lesion progression changes lysophosphatidic acid homeostasis to favor its accumulation. Am J Pathol 176: 3073-3084, 2010.

30. Ghosh HS, McBurney M and Robbins PD: SIRT1 negatively regulates the mammalian target of rapamycin. PLoS One 5: e9199, 2010.

31. Xu J, Dang Y, Ren YR and Liu JO: Cholesterol trafficking is required for mTOR activation in endothelial cells. Proc Nat Acad Sci USA 107: 4764-4769, 2010.
32. Gareus R, Kotsaki E, Xanthoulea S, van der Made I, Gijbels MJ, Kardakaris R, Polykratis A, Kollias G, de Winther MP and Pasparakis M: Endothelial cell-specific NF- $\kappa \mathrm{B}$ inhibition protects mice from atherosclerosis. Cell Metab 8: 372-383, 2008.

33. Ma KL, Ruan XZ, Powis SH, Chen Y, Moorhead JF and Varghese Z: Inflammatory stress exacerbates lipid accumulation in hepatic cells and fatty livers of apolipoprotein E knockout mice. Hepatology 48: 770-781, 2008.

34. Yeung F, Hoberg JE, Ramsey CS, Keller MD, Jones DR, Frye RA and Mayo MW: Modulation of NF- $\mathrm{KB}$-dependent transcription and cell survival by the SIRT1 deacetylase. EMBO J 23: 2369-2380, 2004.

35. Salminen A, Kauppinen A, Suuronen T and Kaarniranta K: SIRT1 longevity factor suppresses NF- $\mathrm{B}$-driven immune responses: Regulation of aging via NF-kappaB acetylation? Bioessays 30: 939-942, 2008.

36. Cohen HY, Miller C, Bitterman KJ, Wall NR, Hekking B, Kessler B, Howitz KT, Gorospe M, de Cabo R and Sinclair DA: Calorie restriction promotes mammalian cell survival by inducing the SIRT1 deacetylase. Sci 305: 390-392, 2004.

37. Blagosklonny MV: Calorie restriction: decelerating mTOR-driven aging from cells to organisms (including humans). Cell Cycle 9: 683-688, 2010.

38. Ma L, Dong W, Wang R, Li Y, Xu B, Zhang J, Zhao Z and Wang Y: Effect of caloric restriction on the SIRT1/mTOR signaling pathways in senile mice. Brain Res Bull 116: 67-72, 2015. 